\title{
FORÇA MUSCULAR RESPIRATÓRIA E MOBILIDADE TORÁCICA EM PORTADORES DE DOENÇA DE PARKINSON
}

\author{
RESPIRATORY MUSCLE STRENGTH AND THORACIC MOBILITY \\ IN PATIENTS WITH PARKINSON'S DISEASE
}

\author{
Cristiane Tasca ${ }^{a^{*}}$, Rodrigo Costa Schuster ${ }^{b^{*}}$, Luiz Fernando Callage Alvarenga ${ }^{\mathrm{c}^{*}}$ \\ aschusterfisio@gmail.com, brodrigo_schuster_fisio@yahoo.com.br, cluiz.alvarenga@fsg.br \\ *Faculdade da Serra Gaúcha - Caxias do Sul (RS), Brasil
}

Data de entrada do artigo: 04/08/2013

Data de aceite do artigo: 03/12/2013

\section{RESUMO}

Introduçáo: A doença de Parkinson é mais frequente em idosos, sua progressão é lenta, produz enfraquecimento do movimento voluntário e rigidez muscular. Com o envelhecimento, o sistema respiratório apresenta alterações estruturais, perda de elasticidade, dilatação alveolar, diminuição do estímulo neural para os músculos respiratórios e alteraçóes de volumes, capacidades e fluxos respiratórios. Objetivos: Verificar a expansibilidade torácica e a força da musculatura respiratória. Materiais e Métodos: Pacientes foram classificados quanto à fase da doença de Parkinson por meio da Escala de Hoehn e Yahr, e foram utilizados como instrumentos de avaliação a cirtometria e a manovacuometria. Resultados: 10 indivíduos com diagnóstico de doença de Parkinson, grande maioria homens (80\%). Quanto ao estadiamento da doença pela Escala de Hoehn e Yahr, 40\% apresentaram-se no estágio 2,5 - doença bilateral leve. Quanto à mobilidade torácica, obtiveram-se médias de inspiração máxima de 96,8 $\pm 10,77$ $\mathrm{cm}$, de expiração máxima de $93,5 \pm 10,93 \mathrm{~cm}$ e a variação entre elas foi de $3,3 \pm 1,41 \mathrm{~cm}$. Quanto à força muscular respiratória, obtiveram-se valores de PImáx, tendo como média nos indivíduos 50,5 $\pm 30,31$ cmH2O, a média prevista para esta amostra foi de $96,4 \pm 13,91 \mathrm{cmH} 2 \mathrm{O}$ e a porcentagem da PImáx prevista de 54,97 $\pm 34,87$. Já em relação PEmáx, a média nos indivíduos parkinsonianos mensurada foi de $6217,02 \pm \mathrm{cmH} 2 \mathrm{O}$, a média prevista para esta amostra foi de $183,4 \pm 27,58 \mathrm{cmH} 2 \mathrm{O}$ e a porcentagem da PEmáx prevista de 32,24 \pm 9,49. Conclusão: Verificou-se que a força muscular respiratória apresentase diminuída e, consequentemente, a mobilidade torácica também é menor nos portadores da doença de Parkinson, quando comparados com valores pré-determinados para indivíduos hígidos.

Palavras-chave: Doença de Parkinson; força muscular; músculos respiratórios.

\section{ABSTRACT}

Introduction: Parkinson's disease is more frequent in the elderly, their progression is slow, produces weakness of voluntary movement and muscle stiffness. With aging, the respiratory system presents structural changes, loss of elasticity, alveolar dilation, decreased neural stimulation to the respiratory muscles and changes in volumes, capacities and respiratory flows. Objectives: Verification of the chest expansion and respiratory muscle strength. Materials and Methods: Patients were classified as to the stage of Parkinson's disease by Hoehn and Yahr and cirtometry and manovacuometry were used as assessment tools. Results: 10 individuals diagnosed with Parkinson's disease, mostly men (80\%). As for the staging of the disease by Hoehn and Yahr, 40\% appeared in stage 2.5 - mild bilateral disease. As for thoracic mobility, we obtained average maximum inspiration of $96.8 \pm 10.77 \mathrm{~cm}$, the maximum expiration of $93.5 \pm 10.93 \mathrm{~cm}$ and the variation between them was $3.3 \pm 1.41 \mathrm{~cm}$. As for the respiratory muscle strength were obtained MIP with the average at $50.5 \pm 30.31 \mathrm{cmH} 2 \mathrm{O}$ on individuals, the average expected for this sample was $96.4 \pm$ 
$13.91 \mathrm{cmH} 2 \mathrm{O}$ and percent predicted MIP $5497 \pm 34.87$. Regarding MEP, the average measured in parkinsonian subjects was $62 \pm 17.02 \mathrm{cmH} 2 \mathrm{O}$, the average expected for this sample was $183.4 \pm 27.58 \mathrm{cmH} 2 \mathrm{O}$ and the percentage of MEP estimated was $32.24 \pm 9.49$. Conclusion: It was verified that the respiratory muscle strength is reduced and consequently the thoracic mobility is also lower in patients with Parkinson's disease compared to predetermined values for healthy individuals.

Keywords: Parkinson's disease; muscle strength; respiratory muscles.

\section{Introdução}

A doença de Parkinson (DP) foi descrita pela primeira vez por James Parkinson, em 1817. É uma patologia de progressão lenta que produz enfraquecimento gradual do movimento voluntário, rigidez muscular e, possivelmente, tremor ${ }^{1}$. O diagnóstico da doença de Parkinson é estabelecido com a presença de quatro principais sinais: tremor de repouso, rigidez muscular, bradicinesia e alteraçôes posturais, acrescidos de assimetria do quadro e da resposta inicial ao uso de levodopa ${ }^{2}$.

A doença de Parkinson é uma doença de característica progressiva e distribuição universal, atingindo todos os grupos étnicos e classes socioeconômicas. Apresenta certa predominância no sexo masculino, acometendo aproximadamente $0,1 \%$ da populaçáo geral e de $1 \%$ a $2 \%$ da populaçáo acima de 65 anos. Com o envelhecimento da populaçáo, ocorre a formação de um novo perfil epidemiológico, trazendo consigo um volume crescente de doenças crônicas e degenerativas como a doença Parkinson, que está entre as doenças neurodegenerativas de maior incidência em pessoas idosas ${ }^{2}$.

$\mathrm{O}$ envelhecimento pode ser um fator de predisposição. A maioria dos indivíduos com doença de Parkinson se encontra na faixa etária geriátrica e apresenta vulnerabilidade a problemas relacionados com a idade, resultando em comprometimento físico, redução da mobilidade e alteraçốes cognitivas, que estão, por vezes, associadas a outras comorbidades que acompanham a progressão da doença. De etiologia controversa, ainda não se definiu uma causa para esta degeneraçáo, tendo sido associada a distúrbios genéticos, estresse oxidativo, exposição a ambientes tóxicos e infecções, podendo ser de natureza multifatorial ${ }^{2}$.

A dinâmica respiratória é afetada pela diminuição da força dos músculos respiratórios: diafragma, peitorais, intercostais. Por esta razão, a singular dificuldade de mobilização das secreçóes respiratórias pode se tornar prognóstico reservado em parkinsonianos, principalmente se não for tratada em seu início e na fase avançada da doença de Parkinson, a mortalidade está seguidamente relacionada à pneumonia ${ }^{3}$.

Um aspecto relevante da disfunçâo respiratória na doença de Parkinson é a força muscular respiratória. Em algumas concepções, a fraqueza da musculatura respiratória, especialmente da musculatura expiratória, pode ocasionar redução da pressão expiratória máxima, menor fluxo expiratório, assim como aumento do volume residual, possivelmente correlacionado ao agravamento da doença $a^{4}$.

A fisioterapia respiratória é conhecida por sua efetividade na melhora dos sintomas e prevenção de complicações respiratórias. A grande maioria de indivíduos com diagnóstico de Parkinson refere dispneia aos médios esforços e dificuldade na mobilização de tronco durante mudanças de postura e pouca mobilidade do gradil costal, principalmente na expiração. Neste contexto, a fisioterapia tem como objetivos diminuir a dispneia, aumentar a eficiência dos músculos respiratórios, maior expansão torácica, relaxamento de musculatura hipertônica e melhora do padrão respiratório 5 .

As perdas físicas decorrentes da doença de Parkinson: a funcionalidade geral dos pacientes, incluindo a função respiratória, tornando importantes intervençóes que avaliem, previnam e tratem estas. Com o envelhecimento, o sistema respiratório apresenta alteraçóes estruturais, perda de elasticidade, dilataçáo alveolar, diminuição do estímulo neural para os músculos respiratórios e alteraçóes de volumes, capacidades e fluxos respiratórios $^{6}$. Esses fatores acentuam ainda mais a morbidade e a mortalidade dos indivíduos portadores de Parkinson. Este estudo teve como objetivo verificar a força muscular respiratória e a mobilidade torácica em portadores da doença de Parkinson.

\section{Materiais e Métodos}

Esta pesquisa caracteriza-se como observacional, do tipo transversal, em que se realizou avaliação da força muscular respiratória e mobilidade do tórax de pacientes portadores de doença de Parkinson. Ela foi autorizada a ser realizada junto à Associação Brasileira de Parkinson - Seccional Serra Gaúcha, situada na cidade de Bento Gonçalves/RS. E foi aprovada sua realização pelo Comitê de Ética em Pesquisa - Círculo Operário Caxiense/Faculdade da Serra Gaúcha sob nº 68.287, por atender às normas da resolução 196/96 do Conselho Nacional da Saúde para pesquisas envolvendo seres humanos.

Após a aprovação desta pesquisa, foram realizadas visitas à Associação Brasileira de Parkinson - Seccional 
Serra Gaúcha, em que todos os participantes cadastrados e que estavam presentes nas atividades da associação nesses dias foram convidados a participar, sendo que deveriam se enquadrar nos critérios de inclusão, que foram: indivíduos de ambos os gêneros com idades mínimas de 60 anos, integrantes da Associação Brasileira de Parkinson - Seccional Serra Gaúcha, e que aceitaram participar da pesquisa pela assinatura do Termo de Consentimento Livre e Esclarecido. Foram excluídos os voluntários que não estavam em condições de serem avaliados por apresentarem algum déficit cognitivo, estarem acamados e/ou apresentarem doenças pulmonares pré-existentes diagnosticadas.

Os dados foram coletados mediante a ficha de avaliaçáo produzida pelos pesquisadores, também foi realizada avaliação do estágio da doença de Parkinson de cada um, por meio da Escala de Hoehn e Yahr Modificada, desenvolvida mais recentemente e que inclui estágios intermediários que classifica a severidade da doença de Parkinson e abrange, essencialmente, medidas globais de sinais e sintomas que permitem classificar o indivíduo quanto ao nível de incapacidade.

Foi ainda realizada avaliação da mobilidade torácica por cirtometria, sendo realizada em ortostase, com fita métrica ao redor do tórax no nível do apêndice xifoide, em expiração normal, na inspiração e na expiração máximas. A cirtometria, ou perimetria toracoabdominal, consiste em um conjunto de medidas das circunferências de tórax e abdômen durante os movimentos respiratórios. Sua finalidade é avaliar a mobilidade/expansibilidade torácica de forma simples e acessível e, para tal, apenas uma fita métrica é necessária ${ }^{7}$.

Finalmente, foi realizada a avaliação da força muscular respiratória, pela manovacuometria (manovacuômetro analógico da marca Comercial Médica ${ }^{\circledR}$ ), em que foi conectado a uma traqueia de plástico de 16 centímetros de comprimento e 2,4 centímetros de diâmetro interno. A extremidade da traqueia foi conectada a um bocal de plástico rígido. As medidas das pressões respiratórias máximas foram realizadas com os indivíduos sentados, utilizando clipe nasal e mantendo um bocal firmemente entre os lábios. Pelo menos duas manobras para aprendizado foram realizadas. A avaliaçáo só era considerada completa quando o indivíduo realizasse três medidas aceitáveis e, dentre estas, um número mínimo de duas reprodutíveis ${ }^{8}$. Foram consideradas aceitáveis manobras sem vazamentos de ar e com sustentaçáo da pressão por pelo menos um segundo. Houve intervalo de um minuto entre as medidas. Para a medida de PImáx, os indivíduos expiraram no bocal até o volume residual e, posteriormente, geraram um esforço inspiratório máximo contra uma via aérea ocluída. Para PEmáx, os indivíduos inspiraram no bocal até a capacidade pulmonar total e, em seguida, um esforço máximo expiratório contra uma via aérea ocluída era gerado ${ }^{8-9}$. Durante essa última medida, os indivíduos seguraram com as mãos a musculatura perioral da face para evitar vazamento e acúmulo de ar na regiấo lateral da cavidade oral ${ }^{8}$.

A análise estatística dos dados baseou-se em medidas de tendência central e de variabilidade (média e desvio padrão). As análises foram realizadas usando o software Statistical Package for the Social Sciences, versão 15.0.

\section{Resultados}

Fizeram parte da amostra estudada 10 indivíduos com diagnóstico de doença de Parkinson, em sua grande maioria homens (80\%), com média de idade de 68,9 $\pm 7,01$ [60-84] anos. Quanto ao estadiamento da doença pela Escala de Hoehn e Yahr entre os indivíduos avaliados, $40 \%$ apresentaram-se no estágio 2,5 - doença bilateral leve, com recuperaçáo no "teste do empurrão" (Tabela 1).

Tabela 1: Estágios da doença de Parkinson por meio da Escala de Hoehn e Yahr

\begin{tabular}{ccc}
\hline Estadiamento da doença & N & Percentual \\
\hline Estágio 2 & 3 indivíduos & $30 \%$ \\
Estágio 2,5 & 4 indivíduos & $40 \%$ \\
Estágio 3 & 2 indivíduos & $20 \%$ \\
Estágio 4 & 1 indivíduo & $10 \%$ \\
\hline
\end{tabular}

Fonte: dados coletados pelos autores.

$\mathrm{Na}$ avaliação da mobilidade torácica, pela cirtometria, obtiveram-se médias de inspiração máxima de 96,8 $\pm 10,77 \mathrm{~cm}$, de expiraçâo máxima de 93,5 $\pm 10,93 \mathrm{~cm}$ e a variação entre inspiração máxima e expiração máxima de 3,3 $\pm 1,41 \mathrm{~cm}$ (Tabela 2).

Tabela 2: Valores da mobilidade torácica

\begin{tabular}{cc}
\hline Variável & Média \pm DP \\
Cirtometria & $96,8 \pm 10,77$ \\
\hline Insp. máx. & $93,5 \pm 10,93$ \\
Exp. máx. & $3,3 \pm 1,41$ \\
Variação & \\
\hline
\end{tabular}

Fonte: dados coletados pelos autores.

$\mathrm{Na}$ avaliação da força muscular respiratória, por manovacuometria, obtiveram-se valores de PImáx, tendo como média nos indivíduos parkinsonianos 50,5 \pm $30,31 \mathrm{cmH} 2 \mathrm{O}$, a média prevista para essa amostra foi de 96,4 $\pm 13,91 \mathrm{cmH} 2 \mathrm{O}$ e a porcentagem da PImáx prevista de 54,97 $\pm 34,87$. Já em relação PEmáx, a média nos indivíduos parkinsonianos mensurada foi de 62 $\pm 17,02 \mathrm{cmH} 2 \mathrm{O}$, a média prevista para essa amostra foi 
de $183,4 \pm 27,58 \mathrm{cmH} 2 \mathrm{O}$ e a porcentagem da PEmáx prevista de 32,24 \pm 9,49 (Tabela 3).

Tabela 3: Valores da força muscular respiratória - PImáx e PEmáx

\begin{tabular}{cc}
\hline Variável & Média \pm DP \\
\hline Pressáo inspiratória & \\
Medida & $50,5 \pm 30,31$ \\
Prevista & $96,4 \pm 13,91$ \\
\% prevista & $54,97 \pm 34,87$ \\
Pressáo expiratória & \\
Medida & $62 \pm 17,02$ \\
Prevista & $183,4 \pm 27,58$ \\
\% prevista & $34,24 \pm 9,49$ \\
\hline
\end{tabular}

Fonte: dados coletados pelos autores.

\section{Discussão}

A etiologia das disfunçóes respiratórias permanece desconhecida, sendo considerada multifatorial, possivelmente em virtude da fisiopatologia complexa, dos poucos estudos investigativos e, ainda, pelo fato de que as suas repercussôes podem ser assintomáticas. Apesar da etiologia desconhecida, alguns autores sugerem hipóteses acerca da disfunçáo respiratória na doença de Parkinson e suas consequências no dia a dia do parkinsoniano.

Nos resultados encontrados na tabela 1, 40\% dos indivíduos avaliados apresentaram-se no estágio $2,5 \mathrm{da}$ Escala de Hohen e Yahr, que corresponde à doença bilateral leve, ao teste apresentam recuperação no teste do empurrão. Seguido de $30 \%$ na escala 2 , que mostra a doença bilateral sem déficit de equilíbrio. Em seguida, temos os $20 \%$ correspondentes à escala 3 , que expóe a doença bilateral leve a moderada, com alguma instabilidade postural e capacidade para viver independente. Apenas $10 \%$ permanecem na escala 4, que indica a incapacidade grave, ainda capaz de caminhar ou permanecer de pé sem ajuda.

Para Rasr ${ }^{10}$, a Escala de Hohen e Yahr avalia o paciente levando em consideraçóes os reflexos posturais assim como a bilateralidade da doença, especialmente no que se refere à independência para as atividades de vida diária.

Haase, Machado e Oliveira ${ }^{11}$ investigaram os efeitos da fraqueza muscular respiratória no dia a dia, qualidade de vida, nível de atividades e capacidade física de indivíduos com doença de Parkinson - estágios iniciais, em comparação com um grupo controle. O grupo de estudo foi constituído por 66 parkinsonianos e o controle por 32 indivíduos. Os parkinsonianos tiveram suas pressões respiratórias verificadas e estes achados foram correlacionados aos resultados do Índice de Barthel (função de vida diária), Questionário Modificado de Baecke (nível de atividades), Questionário de Qualidade de Vida na Doença de Parkinson, consumo máximo de oxigênio, concentração sanguínea de lactato, frequência cardíaca de pico e número de ciclos completos no teste cicloergômetro. Como resultados, as pressóes respiratórias foram significativamente menores nos parkinsonianos, nos estágios I, II e III de Hohen e Yahr (1967), mas não influenciaram as medidas de avaliaçáo da vida diária, nível de atividades, nem qualidade de vida. Além desse resultado, os autores sugerem que a força muscular respiratória tende a ser um fator interferente no desempenho do exercício físico, de maior repercussão, à medida que os estágios da doença progridem.

Para a avaliação da mobilidade torácica, utilizamos a técnica de cirtometria toracoabdominal, a qual teve a sua precisão e acurácia testadas nos estudos de Caldeira et al. ${ }^{7}$ e Malaguti et al. ${ }^{12}$.

A avaliação do tórax e do abdômen é composta pelo exame físico e pode ser específica com o objetivo de determinar disfunçóes toracoabdominais, como a avaliação da mobilidade torácica, que tem relaçáo direta com a expansibilidade pulmonar, sendo mensurada por meio de técnicas específicas como a cirtometria ou perimetria toracoabdominal - técnica que consiste em um conjunto de medidas das circunferências de tórax e abdômen durante os movimentos respiratórios, sendo um método simples e acessível ${ }^{13-14}$.

Em um estudo de Cardoso e Pereira ${ }^{6}$, as medidas da cirtometria são significativamente menores em indivíduos parkinsonianos, caracterizando acentuada diminuição da sua mobilidade torácica durante a respiraçấo e repercutindo no aumento do trabalho muscular, com consequente diminuição da expansibilidade pulmonar na inspiração e da depressão torácica na expiração. Um tórax rígido e resistente aos movimentos rápidos acarreta a limitação progressiva da ventilação, a fixação postural em flexão e a rigidez da musculatura intercostal o que compromete a mobilidade da caixa torácica.

Carvalho ${ }^{15}$ cita que os valores para a cirtometria considerados normais seriam de 6 a 7 centímetros e que as medidas entre 3 e 4 centímetros corresponderiam a uma capacidade pulmonar 20\% abaixo do normal. Já Bethlem ${ }^{16}$ cita que o coeficiente respiratório pode variar de 5 a 11 centímetros, demonstrando que, embora não exista um consenso sobre esses dados na literatura, o valor encontrado para pacientes parkinsonianos encontra-se abaixo desse valor.

Para Presto e Damázio ${ }^{17}$, a fraqueza muscular é a redução da força muscular em determinado período de tempo, já que a lesão muscular é a redução reversível ou irreversível na contratilidade muscular, que geralmente 
não se resolve com pequenos períodos de repouso. Para se reverter um quadro de fraqueza muscular, deve-se fortalecer os músculos e, para tanto, é necessário que sejam impostas cargas e nutridos adequadamente.

Outro aspecto relevante da disfunçáo respiratória na doença de Parkinson é a força muscular respiratória. $\mathrm{Na}$ concepção de Saleen, Sapienza e Okun ${ }^{18}$, a fraqueza da musculatura respiratória, especialmente da expiratória, pode ocasionar redução da pressão expiratória máxima, menores fluxos expiratórios, assim como aumento do volume residual, sendo possivelmente correlacionada ao agravamento da doença.

Existem discordâncias na literatura em relação à presença de redução da força muscular inspiratória e expiratória em pacientes com DP. Em estudo que avaliou a força muscular respiratória de 40 pacientes com DP, não foram observadas alterações desta função ${ }^{6}$. Alves, Coelho e Brunetto ${ }^{5}$ defendem que a redução da PImáx pode estar relacionada à fadiga muscular e ao comprometimento neurológico desses pacientes.

Além das alteraçóes ventilatórias secundárias às manifestaçóes neurológicas da DP, o tratamento à base de levodopa gera obstrução das vias aéreas superiores, diminuição da complacência da caixa torácica e discinesias musculares produzindo dispneia, hipoventilação, atelectasia e retenção de secreçóes pulmonares 5 .

Embora esteja claro que as alteraçóes ventilatórias severas são frequentes nos pacientes com DP, muitos destes não relatam sintomas respiratórios, o que pode ser justificado pelo sedentarismo adotado pela maioria desses pacientes. Em função da tendência a pouca atividade física, não há solicitação de esforço suficiente para provocar as manifestações de disfunçôes respiratórias?.

Existe uma grande variação no que diz respeito a valores de pressões respiratórias máximas descritas na literatura para pacientes com DP. No estudo de Silva et al. ${ }^{19}$, os valores da PEmáx de portadores de DP, com tempo de evoluçáo da doença de até cinco anos, não apresentaram diferença quando comparados a um grupo controle composto por indivíduos saudáveis. Contudo, o autor encontrou diminuição significativa deste parâmetro quando os pacientes com DP apresentavam mais de cinco anos de evolução da doença, justificando este fato com a gravidade do quadro neurológico. No presente estudo não foi levado em consideração o tempo de evolução da DP, mas constatou-se que quanto maior o comprometimento funcional, mais reduzidas as pressôes expiratórias máximas.

Sabaté et al. ${ }^{20}$ também encontraram valores menores para PEmáx em um grupo de pacientes com DP com idade média de 67,7 anos, quando comparados a um grupo de não parkinsonianos. Brown ${ }^{21}$ enfatiza que na DP, durante o esforço expiratório há dificuldade de contração muscular rápida e manutenção da atividade da musculatura intercostal, justificando assim a diminuição da PEmáx em portadores de DP. Alves, Coelho e Brunetto 5 , em estudo que analisou a função ventilatória de um indivíduo com DP de 47 anos, encontraram, antes da aplicação de tratamento fisioterapêutico, valor de PImáx referente a $53 \%$ do predito, atribuindo isto ao comprometimento da coordenação muscular durante realização das manobras específicas da manovacuometria, ou ainda, devido à fadiga muscular ou gravidade do quadro neurológico. Silva et al. ${ }^{19}$ relatam comprometimento das pressôes expiratórias máximas em pacientes com cinco a dez anos de tempo de diagnóstico. Por outro lado, em estudo que comparou a força muscular inspiratória e expiratória de 40 pacientes com DP com outros 40 não parkinsonianos, não encontrou diferença significativa, independente do tempo de evolução da doença ${ }^{6-9}$.

\section{Conclusão}

A partir dos resultados do presente estudo, pôde-se verificar que a força muscular respiratória apresentase diminuída e, consequentemente, a mobilidade torácica também é menor nos portadores da doença de Parkinson quando comparados com valores pré-determinados para indivíduos hígidos.

Baseado nesses resultados, podemos pensar em programas de prevenção a complicaçóes respiratórias para os portadores de doença de Parkinson, fazendo com que evite ou minimize o surgimento de infecçôes respiratórias neste público, o que muitas vezes pode levar até ao seu óbito.

\section{Referências}

1. Thonson A, Skinner A, Piercy J. Fisioterapia de TIDY. São Paulo: Livraria Santos, 2000.

2. Gonçalves L, Alvares M, Arruda M. Pacientes Portadores de Parkinson: significado das suas vivências. Acta Paul Enferm. 2007; 20(1): 62-8.

3. Reis T. Doença de Parkinson: pacientes, familiares e cuidadores. Porto Alegre: Pallotti; 2004.

4. Ferreira FV, Cielo CA, Trevisan ME. Força muscular respiratória, postura corporal, intensidade vocal e tempos máximos de fonação na doença de Parkinson. Rev CEFAC. 2012; 14(2): 361-8.

5. Alves LA, Coelho CA, Brunetto FA. Fisioterapia respiratória na doença de Parkinson idiopática: relato de caso. Rev Fisioter Pesq. 2005; 12(3): 46-9.

6. Cardoso SRX, Pereira JS. Análise da função respiratória na doença de Parkinson. Arq Neuropsiquiatr. 2002; 60(1): 91-5. 
7. Caldeira VS, Starling CCD, Brito RR, Martins JA, Sampaio RF, Parreira VF. Precisão e acurácia da cirtometria em adultos saudáveis. J Bras Pneumol. 2007; 33(5): 519-26.

8. Souza RB. Pressóes respiratórias estáticas máximas. J Pneumol. 2002; 28(3): 155-65.

9. Parreira VF, Guedes LU, Quintâo DG, Silveira EP, Tomich GM, Sampaio RF et al. Padrão respiratório em pacientes portadores da doença de Parkinson e em idosos assintomáticos. Rev Acta Fisiátr. 2003; 10(2): 61-6.

10. Pinto RASR, Borges V, Aguiar PMC, Ferraz FAP, Hisatugo MK, Ferraz HB. Avaliação das atividades da vida diária de pacientes com doença de Parkinson submetidos à cirurgia estereotáxica. Arq Neuropsiq. 2002; 60(2B): 435-41.

11. Haase DCBV, Machado DC, Oliveira JGD. Atuação da fisioterapia no paciente com doença de Parkinson. Rev Fisioter Mov. 2008; 21(1): 79-85.

12. Malaguti C, Rondelli RR, Souza LM, Domingues M, Dal Corso S. Reliability of chest wall mobility and its correlation with pulmonary function in patients with chronic obstructive pulmonary disease. Respiratory Care. 2009; 54(12): 1703-11.

13. Paulin E, Brunetto AF, Carvalho CRF. Efeitos de programa de exercícios físicos direcionado ao aumento da mobilidade torácica em pacientes portadores de doença pulmonar obstrutiva crônica. J Pneumol. 2003; 29(5): 287-94.
14. Moreno MA, Silva E, Zuttin R, Gonçalves M. Efeito de um programa de treinamento de facilitaçáo neuromuscular proprioceptiva sobre a mobilidade torácica. Fisioter Pesq. 2009; 16(2): 161-5.

15. Carvalho A. Semiologia em reabilitação. São Paulo: Atheneu; 1994.

16. Bethlem N. Pneumologia. 4 ed. São Paulo: Atheneu; 1995.

17. Presto B, Damázio L. Fisioterapia respiratória. Rio de Janeiro: Elsevier; 2009.

18. Saleen AF, Sapienza CM, Okun MS. Respiratory muscle strength training: treatment and response duration in a patient with early idiophatic Parkinson's disease. NeuroRehabilitation. 2005; 20: 323-33.

19. Silva FS, Pabis JVPC, Alencar AG, Silva KB, NavarroPeternella FM. Evoluçáo da doença de Parkinson e comprometimento da qualidade de vida. Rev Neurocienc. 2010; 18(4): 463-8.

20. Sabaté M, Rodríguez M, Méndez E, Enríquez E, González I. Obstructive and restrictive pulmonary dysfunction increases disability in Parkinson disease. Arch Phys Med Rehabil. 1996; 77(1): 29-34.

21. Brown LK. Respiratory dysfunction in Parkinson's disease. Chest Med 1994; 15(4): 715-27. 